\title{
Efeito da Densidade do Fluxo e da presença de aditivos na Soldagem ATIG de Aço Inoxidável Austenítico
}

\section{(Effect of Flux Density and Addictive Presence on ATIG Welding of Austenitic Stainless Steel)}

\author{
Paulo J. Modenesi ${ }^{1}$, Pedro Colen Neto ${ }^{2}$,Eustáquio Roberto Apolinário ${ }^{3}$, Kássia Batista Dias ${ }^{3}$ \\ ${ }^{1}$ Universidade Federal de Minas Gerais, Belo Horizonte, MG, Brasil, modenesi@demet.ufmg.br \\ ${ }^{2}$ Instituto Federal de Educação, Ciência e Tecnologia Minas Gerais, Betim, MG, Brasil, pedro.colen@ifmg.edu.br \\ ${ }^{3}$ Universidade Federal de Minas Gerais, Belo Horizonte, $M G$, Brasil
}

\begin{abstract}
Resumo
A soldagem ATIG é uma variante simples do processo TIG convencional que permite aumentar a penetração da solda e, por exemplo, possibilitar a soldagem em um passe, com penetração total e sem abertura de chanfro, para juntas com espessura de $5 \mathrm{~mm}$ ou mais. Diferentes mecanismos foram propostos para explicar este efeito com destaque para o da contração do arco pela presença de íons negativos e o da alteração no movimento de metal líquido na poça de fusão associada com variações da tensão superficial em função da temperatura. O presente trabalho avalia o efeito da quantidade de um fluxo de um componente (óxido de cromo III, $\mathrm{Cr}_{2} \mathrm{O}_{3}$ ) colocado na superficie da peça e de adições de $\mathrm{KClO}_{4}$ e $\mathrm{Al}_{2} \mathrm{O}_{3}$ no formato do cordão de solda. Foram realizados três conjuntos de experimentos consistindo na deposição de cordões sobre chapa de aço inoxidável austenítico ABNT 304 com $5 \mathrm{~mm}$ de espessura. No primeiro conjunto, variou-se a quantidade de fluxo usada, no segundo, estudou-se o efeito de adições de $\mathrm{KClO}_{4}$ e, no terceiro, de $\mathrm{Al}_{2} \mathrm{O}_{3}$. Foram medidos os sinais elétricos (corrente e tensão) durante a soldagem e, em seções transversais dos cordões, a largura, a penetração e a área do cordão. Os resultados indicaram uma pequena variação na tensão de soldagem (aumento inferior a $1 \mathrm{~V}$ ) na passagem da soldagem TIG para a ATIG. A concentração superficial de fluxo afetou a penetração da solda, ocorrendo um aumento brusco de penetração e área do cordão para concentrações entre 7,5 e $15 \mathrm{~g} / \mathrm{m}^{2}$, seguido por uma variação mais suave para concentrações maiores (até $120 \mathrm{~g} / \mathrm{m}^{2}$ ). Por outro lado, enquanto a adição de $\mathrm{KClO}_{4}$, claramente reduziu o aumento de penetração causado pelo fluxo, a adição de $\mathrm{Al}_{2} \mathrm{O}_{3}$ teve um efeito menos significativo no processo.
\end{abstract}

Palavras-chave: Fluxo, formato do cordão, aço inoxidável, soldagem GTAW.

\begin{abstract}
Active flux TIG (ATIG) welding is a simple variant of conventional TIG welding that allows a major improvement in weld bead penetration. Different mechanisms have been proposed to explain the effect of flux. The most accepted ones consider the arc contraction by negative ions vaporized from the flux and liquid metal flow alterations in the weld pool caused by changes the surface tension values. This paper evaluates the effect of one component $\left(\mathrm{Cr}_{2} \mathrm{O}_{3}\right)$ flux concentration and additions of $\mathrm{KClO}_{4}$ and $\mathrm{Al}_{2} \mathrm{O}_{3}$ on ATIG welding bead shape. Three sets of bead-on-plate weld tests were performed on $5 \mathrm{~mm}$ thick AISI 304 steel plates. Electric current and voltage were measured during each welding trial and the resulting bead geometry was evaluated in cross sections of the weld. Results indicated only minor variations in voltage during the transition from TIG to ATIG welding. Surface flux concentration affected weld bead penetration, and maximum penetration was obtained with flux densities between 15 and $60 \mathrm{~g} / \mathrm{m}^{2}$. On the other hand, the addition of $K C L O_{4}$, despite this being a strong oxidizer, reduced weld penetration. A similar effect was linked to additions of $\mathrm{Al}_{2} \mathrm{O}_{3}$ to the flux.
\end{abstract}

Key-words: Flux, weld bead shape, stainless steel, GTA welding.

\section{Introdução}

O processo de soldagem TIG (ou GTAW) é usado na soldagem de ligas mais sofisticadas e quando um bom acabamento e uma elevada qualidade das soldas são requeridos. Neste processo, um arco elétrico é formado entre um eletrodo de tungstênio e a peça

Recebido em 02/10/2012. Texto final em 03/02/2013. e é protegido por um gás ou mistura de gases inertes. O eletrodo de tungstênio é aquecido a elevadas temperaturas, sendo, na maioria das aplicações o responsável pela emissão dos elétrons necessários ao funcionamento do arco.

As principais desvantagens do processo TIG incluem: (a) limitação quanto à máxima espessura soldável, particularmente em operações que tiverem de ser executadas em um único passe, (b) elevada sensibilidade do formato do cordão a variações da composição química do metal de base e (c) menor taxa de deposição do que as taxas usualmente obtidas com processos de soldagem com eletrodo consumível.

Normalmente, nas operações de soldagem TIG de um aço 
inoxidável com atmosfera de proteção de argônio, a penetração está restrita a uma profundidade máxima de $3 \mathrm{~mm}$, com velocidade de soldagem relativamente baixa. A penetração pode ser aumentada de 1 a $2 \mathrm{~mm}$, portanto, de forma limitada, quando o hélio ou hidrogênio são usados como parte da mistura de proteção [1-2], embora, a velocidade de soldagem possa ser substancialmente aumentada (de até $160 \%$ e $90 \%$, respectivamente) [2]. A capacidade de melhorar a penetração através do gás de proteção é ainda limitada pela necessidade de se usar gases inertes ou ligeiramente redutores na soldagem TIG. Desta forma, a seleção fica restrita basicamente a misturas de $\mathrm{Ar}$ com $\mathrm{He}$ e $\mathrm{H}_{2}$.

Por outro lado, o processo de soldagem a plasma (PAW) pode ser usado de modo alternativo ao processo TIG, permitindo efetuar soldas em um único passe em juntas de espessuras acima de $10 \mathrm{~mm}$ [3]. Contudo, esse processo é mais complexo operacionalmente e tem um maior custo inicial.

Uma variante do processo de soldagem TIG, que utiliza fluxos ativos (Active Flux TIG Welding ou ATIG), foi concebida (na década de 60) para soldagem de titânio pelo Paton Institute of Electric Welding, em Kiev, Ucrânia [1,4]. Esta possibilita superar as limitações relativas à baixa penetração e à sensibilidade da penetração à composição química do material de base do processo TIG. Por este processo, placas com espessura de $6 \mathrm{~mm}$ ou mais poderiam ser soldadas em um único passe, sem abertura de chanfro, possibilitando um grande aumento na produtividade [1].

O processo de soldagem ATIG é uma variante simples do processo TIG, não requerendo basicamente nenhum equipamento adicional. Caracteriza-se pela aplicação de uma fina camada de um fluxo sobre a superfície do metal de base na região que será soldada. O fluxo é formado por uma mistura de pós (em torno de 400\#) formados principalmente por óxidos e fluoretos em suspensão em acetona, metanol ou etanol [1, 5-6]. Após a aplicação do fluxo sobre a junta, o líquido volátil é vaporizado, deixando uma camada do fluxo sobre a qual a soldagem é realizada.

Os ingredientes usados na elaboração de fluxos para o processo ATIG são similares aos usados para produzir fluxos para os processos de soldagem com eletrodos revestidos ou ao arco submerso. Muitos dos trabalhos técnicos, possivelmente, por interesses comerciais, não apresenta a composição dos fluxos usados na soldagem ATIG e, frequentemente, estes fluxos têm composição complexa, dificultando a análise da função de seus componentes. Por outro lado Modenesi e colaboradores [7] mostraram que fluxos muito simples, de apenas um componente, podem causar um aumento de penetração, em relação à soldagem TIG convencional, de mais de $100 \%$. Diversos compostos, incluindo a sílica, o rutilo e o óxido de cromo, apresentam capacidade de aumentar fortemente a penetração do cordão de solda neste processo.

Existem, atualmente, diversos mecanismos propostos para explicar o efeito ATIG. Segundo trabalhos publicados principalmente em países da Europa oriental [1, 6, 8-10], componentes do fluxo vaporizados pelo arco elétrico, no processo de soldagem ATIG, causariam uma ação de constrição neste, similar à causada pelo bocal constritor no processo de soldagem a plasma. A densidade de corrente e a força do arco atuando na poça de fusão seriam, desta forma, aumentados e, assim, a penetração ou a velocidade de soldagem também poderiam ser muito aumentadas em comparação com a soldagem TIG convencional, para o mesmo nível da corrente. $\mathrm{O}$ efeito de constrição seria causado por elementos eletronegativos (particularmente, flúor, cloro e oxigênio) que, nas camadas mais externas do arco, tenderiam a capturar elétrons livres, reduzindo, desta forma, a condutibilidade elétrica nestas regiões e aumentando a densidade de corrente nas regiões mais internas do arco.

Um segundo mecanismo associa o aumento de penetração na soldagem ATIG com uma mudança no sentido de movimentação do metal líquido na poça de fusão [11-12]. Esta mudança direcionaria o líquido mais aquecido, localizado sob o arco para o fundo da poça, aumentando a transferência de calor para esta região. Ela seria causada por alterações na forma de variação da tensão superficial do metal líquido ao longo da superfície da poça, resultante possivelmente da transferência de oxigênio (ou outros elementos tensoativos) da escória gerada pelo fluxo para o metal líquido. Este é, atualmente, o mecanismo mais aceito para explicar o aumento da penetração na soldagem ATIG e parece ser confirmado, por exemplo, pela obtenção de um efeito similar na soldagem TIG com gases de proteção levemente oxidantes. Mecanismos adicionais são ainda citados, incluindo a fixação do arco em pontos de escória e o efeito da camada de fluxo como isolante elétrico e térmico [13].

$\mathrm{Lu}$ e colaboradores [14] avaliaram o efeito da quantidade superficial de fluxos de um componente $\left(\mathrm{Cu}_{2} \mathrm{O}, \mathrm{NiO}, \mathrm{SiO}_{2}\right.$ e $\mathrm{TiO}_{2}$ ) no formato do cordão e na concentração de oxigênio no metal de solda em testes realizados em chapas de aço SUS304 com $10 \mathrm{~mm}$ de espessura. Foi observado um aumento no teor de oxigênio com a quantidade de fluxo. Por outro lado, a razão entre a penetração e a largura do cordão tendeu a aumentar com a quantidade de fluxo usada, passando por um máximo e depois se reduzindo.

O presente trabalho busca avaliar, para a soldagem ATIG um fluxo a base de $\mathrm{Cr}_{2} \mathrm{O}_{3}$, o efeito da quantidade aplicada deste fluxo sobre a peça e da presença de dois componentes $\left(\mathrm{Al}_{2} \mathrm{O}_{3}\right.$ e $\left.\mathrm{KClO}_{4}\right)$ nos resultados da soldagem ATIG em um aço inoxidável austenítico, particularmente na tensão de soldagem e no formato do cordão. $\mathrm{O}$ primeiro componente adicional $\left(\mathrm{Al}_{2} \mathrm{O}_{3}\right)$ não tende a afetar o formato do cordão de solda quando usado sozinho como um fluxo para a soldagem ATIG. Por sua vez, o segundo componente é um material que, quando aquecido, possui uma alta capacidade de liberação de oxigênio. Para a sua escolha, considerou-se que este elemento é possivelmente um dos principais responsáveis pelo aumento de penetração na soldagem ATIG.

\section{Materiais e Métodos}

Foram realizados três conjuntos de testes de soldagem TIG sobre chapas de $5 \mathrm{~mm}$ de espessura de aço inoxidável austenítico (ABNT 304). No primeiro conjunto (Tabela 1), avaliou-se o efeito da quantidade de fluxo (de apenas um componente: $\mathrm{Cr}_{2} \mathrm{O}_{3}$ ) 
aplicada na superfície do metal base. No segundo conjunto (Tabela 2), foram consideradas, conjuntamente, a quantidade de fluxo e a mistura a este de $\mathrm{KClO}_{4}$ (perclorato de potássio) e, no terceiro conjunto (Tabela 3 ), avaliou-se o efeito da presença de $\mathrm{Al}_{2} \mathrm{O}_{3}$ em conjunto com $\mathrm{Cr}_{2} \mathrm{O}_{3}$ mantendo-se uma densidade superficial $\left(\mathrm{g} / \mathrm{m}^{2}\right)$ fixa. No segundo conjunto, uma quantidade fixa de perclorato de potássio foi usada, correspondente a $6 \mathrm{~g} / \mathrm{m}^{2}$ em substituição a igual quantidade de $\mathrm{Cr}_{2} \mathrm{O}_{3}$ no fluxo. $\mathrm{O}_{\mathrm{Cr}_{2}} \mathrm{O}_{3}$ foi fornecido por um fabricante de consumíveis de soldagem, com um nível de impurezas inferior a $1 \%$ e tipicamente em torno de $0,4 \%$. $\mathrm{O} \mathrm{KClO}_{4}$ e o $\mathrm{Al}_{2} \mathrm{O}_{3}$ foram obtidos de um laboratório químico com "pureza analítica".

Tabela 1. Projeto experimental do $1^{\circ}$ bloco de testes.

\begin{tabular}{|c|c|c|}
\hline Condição & $\begin{array}{c}\mathrm{Cr}_{2} \mathrm{O}_{3} \\
\left(\mathrm{~g} / \mathrm{m}^{2}\right)\end{array}$ & Número de testes \\
\hline 1 & 7,5 & 2 \\
\hline 2 & 15 & 4 \\
\hline 3 & 30 & 4 \\
\hline 4 & 60 & 4 \\
\hline 5 & 120 & 2 \\
\hline
\end{tabular}

Tabela 2. Projeto experimental do $2^{\circ}$ bloco de testes.

\begin{tabular}{|c|c|c|c|}
\hline Condição & $\begin{array}{c}\mathrm{Cr}_{2} \mathrm{O}_{3} \\
\left(\mathrm{~g} / \mathrm{m}^{2}\right)\end{array}$ & $\begin{array}{c}\mathrm{KClO}_{4} \\
\left(\mathrm{~g} / \mathrm{m}^{2}\right)\end{array}$ & $\begin{array}{c}\text { Número de } \\
\text { testes }\end{array}$ \\
\hline 1 & 9 & 6 & 2 \\
\hline 2 & 24 & 6 & 2 \\
\hline 3 & 54 & 6 & 1 \\
\hline
\end{tabular}

Tabela 3. Projeto experimental do $3^{\circ}$ bloco de testes.

\begin{tabular}{|c|c|c|c|}
\hline Condição & $\begin{array}{c}\mathrm{Cr}_{2} \mathrm{O}_{3} \\
\left(\mathrm{~g} / \mathrm{m}^{2}\right)\end{array}$ & $\begin{array}{c}\mathrm{Al}_{2} \mathrm{O}_{3} \\
\left(\mathrm{~g} / \mathrm{m}^{2}\right)\end{array}$ & Número de testes \\
\hline 1 & 60 & 0 & 1 \\
\hline 2 & 55 & 5 & 1 \\
\hline 3 & 50 & 10 & 2 \\
\hline 4 & 40 & 20 & 2 \\
\hline
\end{tabular}

Em cada conjunto, os testes foram executados em sequência aleatória. Antes de cada teste, um cupom de 150 x $50 \mathrm{~mm}$ do metal base foi limpo com acetona e uma máscara de adesivo com abertura de $1000 \mathrm{~mm}^{2}$ foi colada em sua superfície. Sobre esta abertura, o peso correspondente de fluxo foi espalhado uniformemente com acetona. Após a secagem da acetona, a máscara foi retirada (Figura 1).

$\mathrm{O}$ cupom foi preso em um gabarito de cobre, inicialmente à temperatura ambiente, para a soldagem que foi realizada em um dispositivo mecanizado, com a solda se iniciando sempre no lado do corpo de prova sem fluxo. Este bloco de cobre tende a uniformizar as condições de extração de calor e, em conjunto com a relativamente baixa condutividade térmica dos aços inoxidáveis austeníticos, facilita a obtenção de um regime quase-estacionário do fluxo de calor durante a soldagem. A obtenção destas condições foi confirmada visualmente nos cordões obtidos pela manutenção da uma largura do cordão uniforme ao longo, respectivamente das regiões de soldagem TIG e ATIG dos corpos de prova. As soldas foram feitas sobre chapa, se iniciando na região sem fluxo e tendo um comprimento de aproximadamente $140 \mathrm{~mm}$. Destes, em torno dos $70 \mathrm{~mm}$ finais foram feitos sobre a camada de fluxo (soldagem ATIG). As condições de soldagem foram mantidas fixas e os principais parâmetros usados estão mostrados na tabela 4. Durante a soldagem, os valores de corrente e tensão foram registrados com um sistema digital de aquisição de dados a uma taxa de $10 \mathrm{kHz}$, sendo tirada a média de cada bloco de 500 medidas, cujo valor foi salvo em arquivo, resultando em uma taxa final de aquisição de dados de $20 \mathrm{~Hz}$. Estes dados foram usados para o cálculo da corrente e tensão médias de soldagem e da diferença de tensão entre as condições de soldagem ATIG e TIG $(\Delta U)$. Para o cálculo de $\Delta \mathrm{U}$, as tensões para cada condição de soldagem foram calculadas em intervalos de $5 \mathrm{~s}$ antes e após o arco atingir a camada de fluxo, desconsiderando-se do cálculo um período de aproximadamente $2 \mathrm{a} 3 \mathrm{~s}$, correspondente à passagem do arco entre as regiões sem e com fluxo.

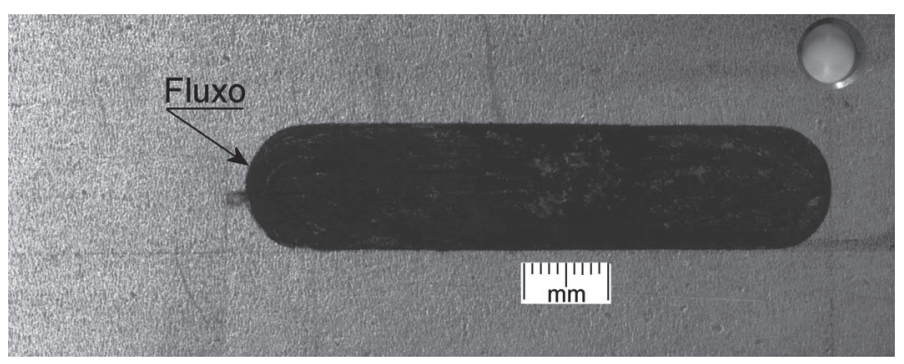

Figura 1. Detalhe da superfície do corpo de prova após a deposição da camada de fluxo com a máscara.

Tabela 4. Condições de soldagem TIG.

\begin{tabular}{|l|l|l|l|}
\hline Eletrodo & EWTh-2 & Gás de proteção: & Argônio \\
\hline Diâmetro: & $3,2 \mathrm{~mm}$ & Vazão: & $10 \mathrm{~L} / \mathrm{min}$ \\
\hline Ângulo da ponta: & $60^{\circ}$ & Comp. de arco: & $3 \mathrm{~mm}$ \\
\hline Corrente: & $200 \mathrm{~A}$ & Polaridade: & CC- \\
\hline Velocidade: & $0,20 \mathrm{~m} / \mathrm{min}$ & Posição: & Plana \\
\hline
\end{tabular}

Foram retiradas, de cada corpo de prova soldado, quatro seções transversais ao cordão, duas na parte correspondente à TIG e duas na parte ATIG. Estas seções foram feitas na região central dos corpos de prova, entre 10 e $25 \mathrm{~mm}$ antes e após a transição das condições de soldagem (de TIG convencional para ATIG). Comparações entre as medidas das dimensões do cordão feitas nas seções consecutivas de uma dada condição de soldagem (TIG ou ATIG) não mostraram uma tendência de variação com a posição de cada seção. Isto segure que as condições de soldagem já haviam atingido um estado quase-estacionário de transferência de calor. As seções foram preparadas para análise macrográfica e atacadas com reativo de cloreto férrico. Elas foram fotografadas em condições padronizadas e as imagens analisadas com o programa ImageJ para medição da largura, penetração e área 
da seção transversal do cordão. Adicionalmente, a largura do cordão foi medida em fotos da superfície dos cordões.

\section{Resultados e Discussão}

A presença do fluxo alterou as condições operacionais de soldagem. Com o fluxo de $\mathrm{Cr}_{2} \mathrm{O}_{3}$ puro, a tensão de soldagem elevou-se ligeiramente em relação à soldagem TIG (Figura 2). Variações similares foram observadas por Perry e Marya [15] na soldagem de titânio com um fluxo a base de fluoretos, e por Modenesi e colaboradores [7], por Shyu e colaboradores [16] e por Kuang-Hung Tseng e Chih-Yu Hsu [17] na soldagem de aço inoxidável com fluxos de diversos componentes, incluindo $\mathrm{Al}_{2} \mathrm{O}_{3}, \mathrm{SiO}_{2}, \mathrm{Cr}_{2} \mathrm{O}_{3}, \mathrm{MnO}_{2}$ e TiO 2 . Em todos os casos, as mudanças são pequenas, um aumento de $1 \mathrm{~V}$ ou menos na tensão. Para um fluxo de $\mathrm{TiO}_{2}$, esta variação pode ser nula ou mesmo negativa [7 e 18]. O aumento da tensão de soldagem foi associado por alguns autores [17, 19-20] com o mecanismo de constrição do arco. Contudo, o valor relativamente pequeno desta variação e a ocorrência de redução da tensão com alguns tipos de fluxo capazes de aumentar a penetração na soldagem ATIG [7] não parecem confirmar esta associação. No presente trabalho, foi observada uma possível correlação entre as variações de tensão e a penetração (ver abaixo), mas, como já indicado, as variações observadas foram relativamente pequenas sugerindo que este efeito tenha um papel secundário.

Nos testes realizados, durante a soldagem ATIG, o processo apresentou indícios de uma menor estabilidade na forma, por exemplo, uma maior variabilidade da tensão de soldagem. Assim, no primeiro bloco de testes o desvio padrão da tensão de soldagem foi, em média, 98\% maior nos intervalos correspondentes à soldagem ATIG em comparação com os valores obtidos durante a soldagem TIG (Figura 2). Esta menor estabilidade pode ser atribuída, em parte a alguma heterogeneidade na distribuição do fluxo, contudo, ela parece ser inerente ao uso do fluxo o que é confirmado por mudanças no aspecto superficial do cordão (maior variação na sua largura e formação de mordeduras).

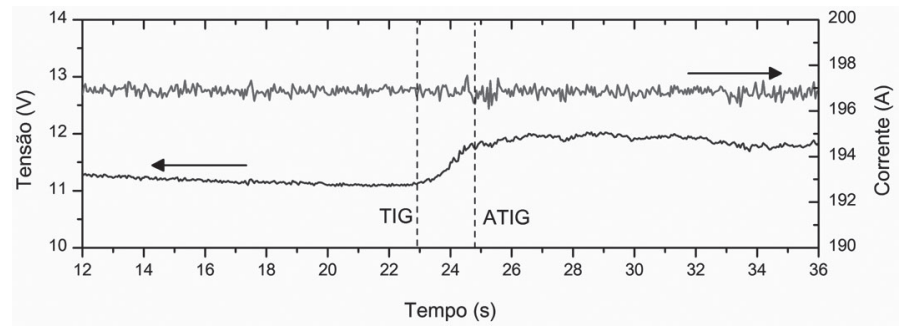

Figura 2. Variações de corrente e tensão na transição entre a soldagem TIG e ATIG. O intervalo entre as linhas pontilhadas marca a transição entre as soldagens TIG e ATIG.

A figura 3 mostra a variação de $\Delta \mathrm{U}\left(=\mathrm{U}_{\text {ATIG }}-\mathrm{U}_{\text {TIG }}\right)$ com a densidade superficial dos fluxos de $\mathrm{Cr}_{2} \mathrm{O}_{3}$ e de $\left(\mathrm{Cr}_{2} \mathrm{O}_{3}+\mathrm{KClO}_{4}\right)$. No primeiro caso, como já colocado, há um aumento na tensão durante a soldagem ATIG que é inferior a $1 \mathrm{~V}$. O valor de $\Delta \mathrm{U}$ atinge um máximo para densidades superficiais de fluxo em torno de $30 \mathrm{~g} / \mathrm{m}^{2}$, se estabilizando ou, possivelmente, se reduzindo levemente para densidades maiores de fluxo. A adição de $\mathrm{KClO}_{4}$ parece restringir o aumento da tensão de soldagem que passa a ocorrer somente quando a quantidade de $\mathrm{Cr}_{2} \mathrm{O}_{3}$ no fluxo atinja um valor suficientemente elevado. Finalmente, embora não mostrado na figura, observou-se que a substituição de $\mathrm{Cr}_{2} \mathrm{O}_{3}$ por $\mathrm{Al}_{2} \mathrm{O}_{3}$ no fluxo não pareceu alterar, nas quantidades testadas, $\mathrm{o}$ aumento de tensão observado nos testes com fluxo contendo apenas $\mathrm{Cr}_{2} \mathrm{O}_{3}$. Este último componente é comum na composição de fluxos para soldagem ATIG de aços inoxidáveis austeníticos. Diversos trabalhos [7, 16, 21-22] indicam que o $\mathrm{Cr}_{2} \mathrm{O}_{3}$ tem uma capacidade pronunciada de aumento da penetração na soldagem TIG tanto quando usado de forma isolada como em conjunto com outros componentes. $\mathrm{O}_{\mathrm{Cr}_{2}} \mathrm{O}_{3}$ é, em geral, considerado, juntamente com o $\mathrm{SiO}_{2}$ e o $\mathrm{TiO}_{2}$, como um dos componentes mais efetivos para aumentar a penetração na soldagem ATIG de aços inoxidáveis austenítico. Por outro lado, resultados de alguns dos trabalhos já indicados sugerem que a alumina não afeta de forma significativa a penetração na soldagem TIG de aços inoxidáveis austeníticos. Finalmente, com relação ao perclorato de potássio $\left(\mathrm{KClO}_{4}\right)$, não foram encontradas indicações de sua aplicação como um componente de fluxo para a soldagem ATIG.

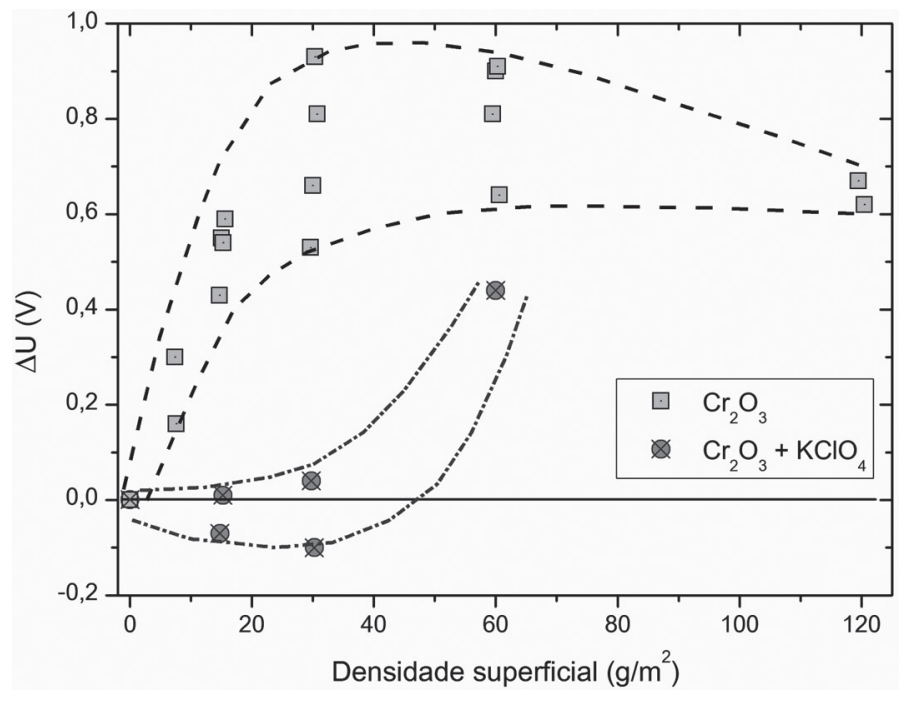

Figura 3. Variações da tensão entre as soldagens ATIG e TIG $(\Delta \mathrm{U})$ em função da densidade superficial de fluxo $\left(1^{\circ}\right.$ e $2^{\circ}$ blocos de testes).

O perclorato de potássio é um composto altamente oxidante que tende a se decompor em torno de $600^{\circ} \mathrm{C}$ e liberar oxigênio pela reação:

$$
\mathrm{KClO}_{4} \rightarrow \mathrm{KCl}+2 \mathrm{O}_{2}
$$

Assim, caso parte do oxigênio gerado fosse transferido para a poça de fusão, este componente poderia aumentar a capacidade do fluxo, com uma dada densidade superficial, afetar a penetração da solda (considerando a reversão da direção das correntes de metal líquido na poça de fusão como o mecanismo responsável pelo aumento da penetração na soldagem ATIG).

A figura 4 mostra o aspecto superficial de um cordão de solda na região de transição entre as condições de soldagem TIG e 
ATIG. Pode-se observar a maior largura, maior regularidade e limpeza (ausência de resíduos) do cordão obtido sem o uso de fluxo. Por outro lado, o conjunto dos resultados obtidos indica que a largura e o aspecto superficial dos cordões obtidos com a aplicação de fluxo parecem ser pouco afetados pelas composições e densidades mostradas.

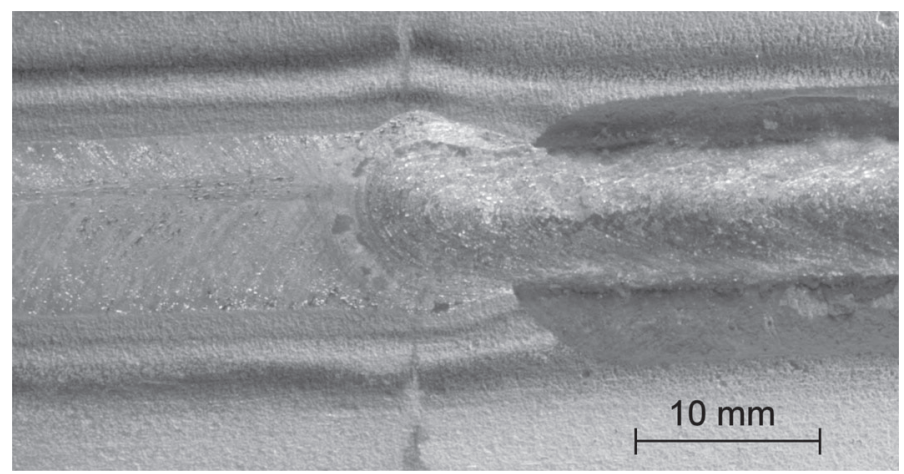

Figura 4. Superfície do cordão de um teste na região de transição entre as soldagens TIG (direita) e ATIG (esquerda). Fluxo usado: $\mathrm{Cr}_{2} \mathrm{O}_{3}$.

A evolução da largura do cordão em função da densidade de fluxo, para os testes com fluxo contendo apenas $\mathrm{Cr}_{2} \mathrm{O}_{3}$, é mostrada na figura 5. Nota-se que a variação observada é similar, embora ocorrendo em sentido contrário, à observada para a tensão de soldagem (Figura 3). Comportamento similar, contudo, não é observado para o fluxo misto $\left(\mathrm{Cr}_{2} \mathrm{O}_{3}+\mathrm{KClO}_{4}\right)$ que reduz a largura do cordão independentemente da variação de tensão observada na transição da soldagem TIG convencional para a soldagem ATIG. Com o terceiro fluxo $\left(\mathrm{Cr}_{2} \mathrm{O}_{3}+\mathrm{Al}_{2} \mathrm{O}_{3}\right)$, a largura do cordão foi similar à obtida com o fluxo simples usado com a mesma densidade superficial $\left(60 \mathrm{~g} / \mathrm{m}^{2}\right)$, independentemente da participação da alumina. Assim esta confirma a sua pouca efetividade como um componente de fluxo para a soldagem ATIG, pelo menos, com relação à largura do cordão.

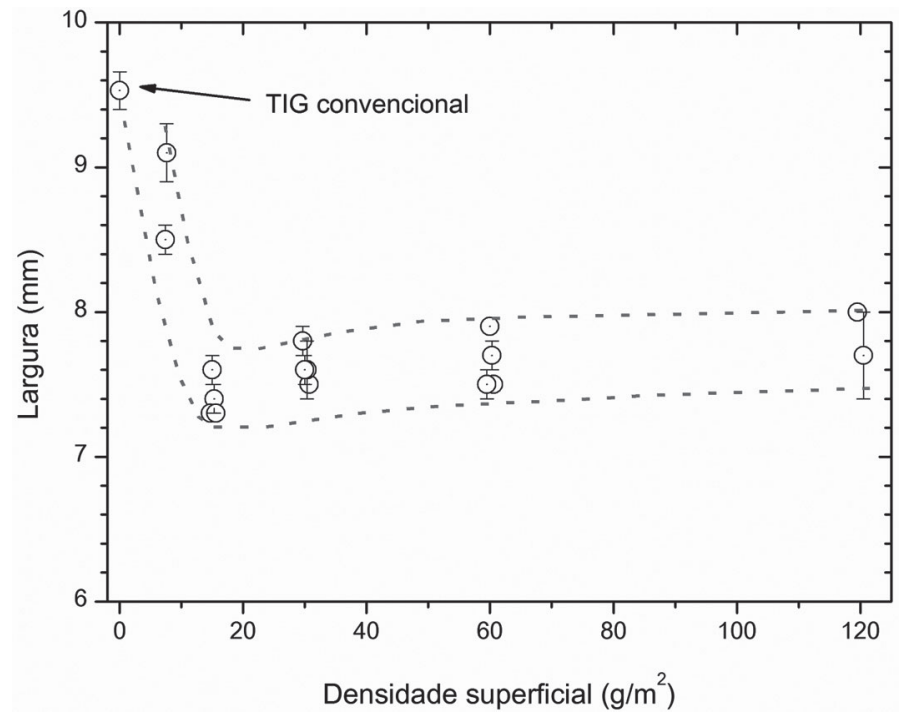

Figura 5. Variação da largura do cordão em função da densidade superficial de aplicação do fluxo de $\mathrm{Cr}_{2} \mathrm{O}_{3}$. A densidade nula corresponde à soldagem TIG convencional.
A figura 6 mostra a evolução da penetração do cordão de solda em função da densidade superficial de fluxo para os testes dos $1^{\circ}$ e $2^{\circ}$ blocos. Em ambos, os resultados indicam um aumento substancial da penetração que passa de 1,6-1,7 mm na soldagem TIG convencional para até a penetração total da chapa usada (com $5 \mathrm{~mm}$ de espessura). No primeiro bloco de testes (figura 7), com fluxo de $\mathrm{Cr}_{2} \mathrm{O}_{3}$, densidades superficiais de $15 \mathrm{~g} / \mathrm{m}^{2}$ já permitiram alcançar penetração total, embora com regiões do cordão ainda apresentando penetração parcial. A penetração total foi conseguida ao longo de todo o cordão para densidades de 30 e $60 \mathrm{~g} / \mathrm{m}^{2}$. Para a maior densidade testada, pequenas regiões de penetração parcial tornaram a ocorrer ao longo do cordão. Este comportamento (um aumento inicial da penetração com a quantidade usada de fluxo até um máximo, seguida por uma redução de penetração para quantidades maiores de fluxo) é similar ao descrito por Lu e colaboradores $[14,23]$ que observaram esta mesma forma de variação na penetração com a com a quantidade de fluxos simples de $\mathrm{Cu}_{2} \mathrm{O}$, $\mathrm{NiO}, \mathrm{SiO}_{2}$ e $\mathrm{TiO}_{2}$.

$\mathrm{Lu}$ e colaboradores $[14,23]$ espalharam quantidades controladas de fluxo (entre $1 \times 10^{-5}$ e $480 \times 10^{-5}$ moles de oxigênio contido no fluxo) em um rebaixo usinado no corpo de prova de 5 x $50 \mathrm{~mm}\left(250 \mathrm{~mm}^{2}\right)$ sobre o qual a solda foi feita, interagindo, em princípio, com todo o material espalhado. Considerando o comprimento do rebaixo, isto correspondeu a uma variação entre $2 \times 10^{-4}$ e 9,6×10-2 moles de [O] por metro de solda. Estes autores observaram um pico de penetração para quantidades de $\mathrm{Cu}_{2} \mathrm{O}, \mathrm{NiO}$ e $\mathrm{SiO}_{2}$ em torno de $50 \times 10^{-5}$ moles de oxigênio contido no fluxo, seguido por uma redução pronunciada já para, por exemplo, $100 \times 10^{-5}$ moles de oxigênio. Para o $\mathrm{TiO}_{2}$, o comportamento foi diferente pois a penetração, após atingir o seu valor máximo, quase não variou. Os autores mediram os teores de oxigênio nas soldas e observaram, para os fluxos de $\mathrm{Cu}_{2} \mathrm{O}, \mathrm{NiO}$ e $\mathrm{SiO}_{2}$, que o máximo de penetração ocorreu para teores na solda em torno de $200 \mathrm{ppm}$.

No presente trabalho, o fluxo foi distribuído sobre uma área que apresentava uma largura maior do que o cordão de solda, não sendo possível uma comparação direta com os trabalhos citados acima, pois, no presente, nem todo o material do fluxo interagia com a poça de fusão. Contudo, considerando a largura média de 7,5 mm para os cordões de solda ATIG e um comprimento médio do cordão de $69 \mathrm{~mm}$ (correspondente ao comprimento da máscara usada), as quantidades correspondentes de fluxo de $\mathrm{Cr}_{2} \mathrm{O}_{3}$ com as densidades de 15 a $60 \mathrm{~g} / \mathrm{m}^{2}$ ficam em torno de $15 \times 10^{-5}$ a $60 \times 10^{-5}$ moles de oxigênio ou $2,1 \times 10^{-3}$ a $8,7 \times 10^{-3} \mathrm{moles} / \mathrm{m}$. Estes valores estão no limite inferior da faixa trabalhada por Lu e colaboradores [23] e dentro das condições em que o aumento de penetração da solda observado por estes autores foi máximo. Deve-se observar, contudo, que, em seus trabalhos, Lu e colaboradores $[14,23]$ não estudaram, como fluxo para soldagem ATIG, o $\mathrm{Cr}_{2} \mathrm{O}_{3}$.

Os resultados (Figura 6) do segundo bloco de testes (com fluxo de $\mathrm{Cr}_{2} \mathrm{O}_{3}+\mathrm{KClO}_{4}$ ) indicam uma menor capacidade de penetração do que no primeiro bloco. Assim, embora regiões de penetração total tenham sido obtidas, estas ocorreram de forma intermitente e em conjunto com seções de penetração parcial.

No terceiro bloco de testes, a penetração do cordão apresentou 


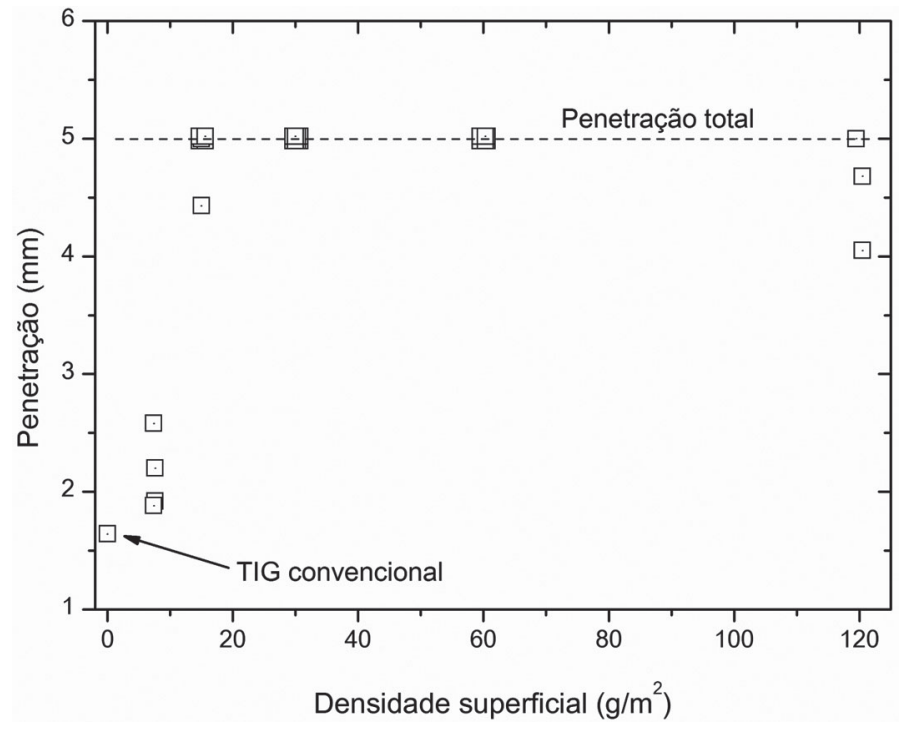

(a)

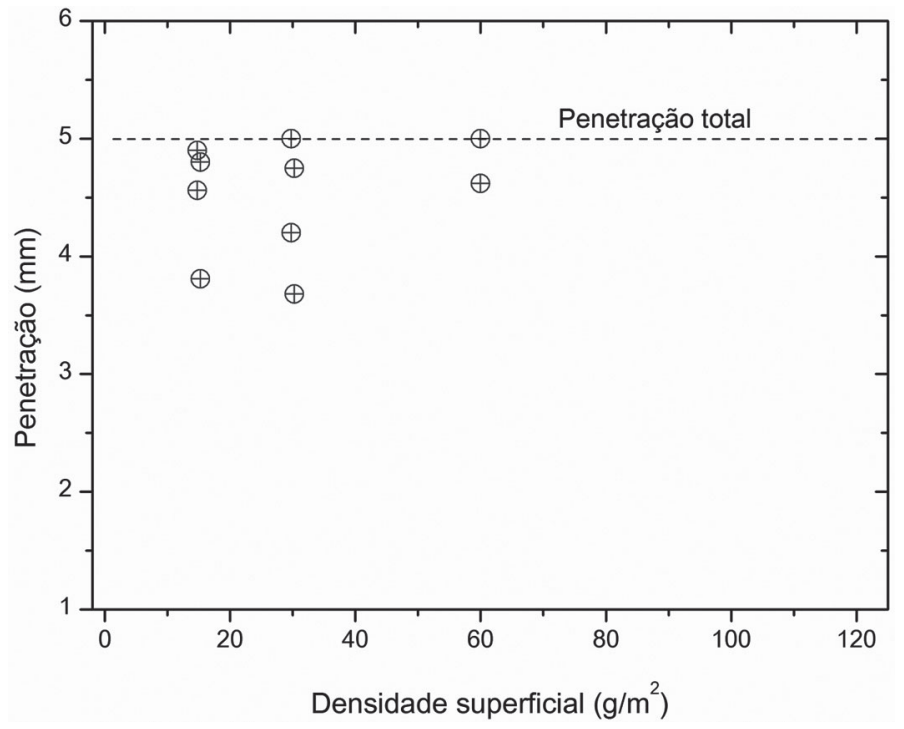

(b)

Figura 6. Variação da penetração do cordão em função da densidade superficial dos fluxos de (a) $\mathrm{Cr}_{2} \mathrm{O}_{3}$ e de (b) $\mathrm{Cr}_{2} \mathrm{O}_{3}+\mathrm{KClO}_{4}$. A densidade nula corresponde à soldagem TIG convencional.

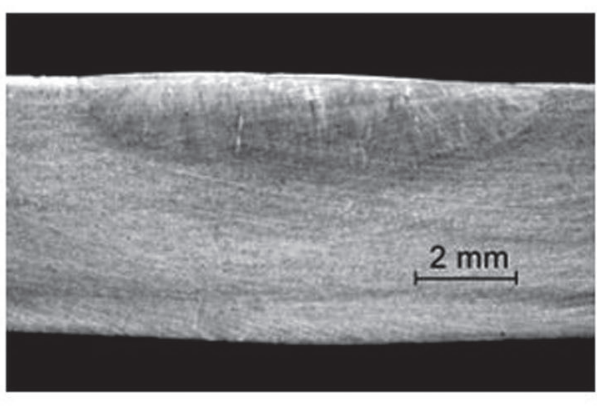

(a)

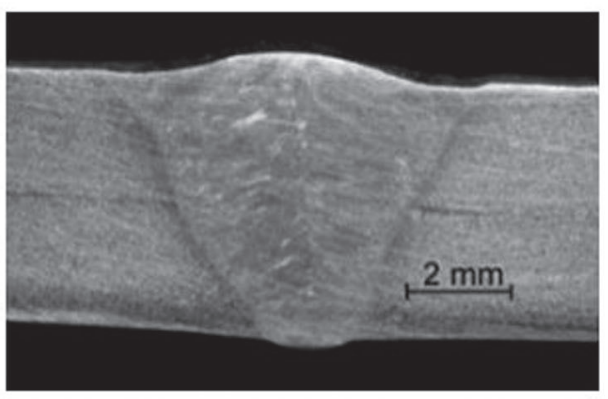

(d)

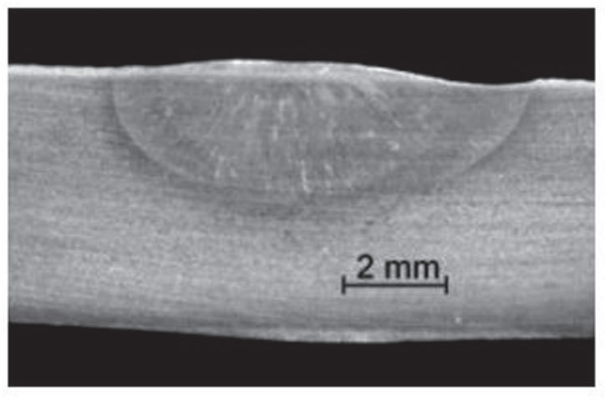

(b)

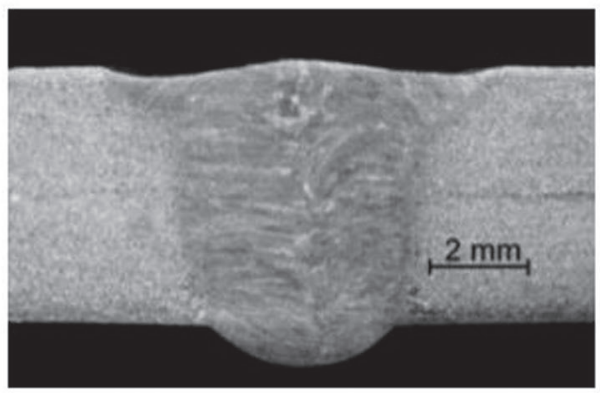

(e)

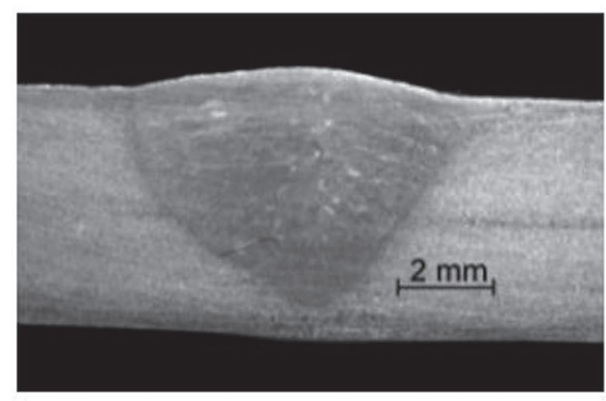

(c)

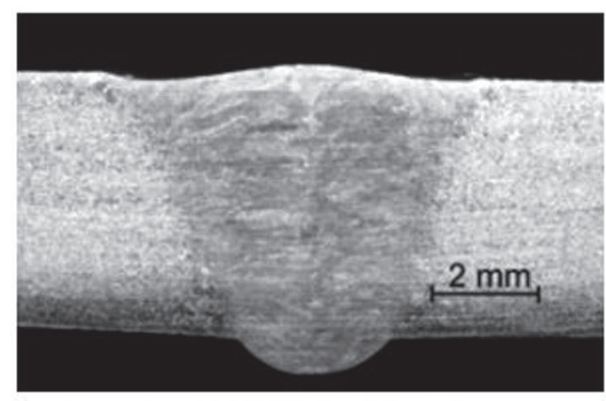

(f)

Figura 7. Exemplos da seção transversal de cordões obtidos no primeiro bloco de testes (fluxo de $\mathrm{Cr}_{2} \mathrm{O}_{3}$ ). (a) TIG, (b) ATIG - 7,5 g/m², (c) ATIG - $15 \mathrm{~g} / \mathrm{m}^{2}$, (d) ATIG - $30 \mathrm{~g} / \mathrm{m}^{2}$, (e) ATIG $-60 \mathrm{~g} / \mathrm{m}^{2}$, (f) ATIG - $120 \mathrm{~g} / \mathrm{m}^{2}$.

um comportamento similar ao observado no segundo bloco (Figura 8). Assim, a substituição do $\mathrm{Cr}_{2} \mathrm{O}_{3}$ pelo $\mathrm{Al}_{2} \mathrm{O}_{3}$ (mantendo a mesma densidade de fluxo, $60 \mathrm{~g} / \mathrm{m}^{2}$ ), reduziu a capacidade de penetração do processo, causando o aparecimento de trechos com penetração parcial na soldagem ATIG.

A figura 9 exibe a variação da área da seção transversal do cordão ("área de fusão") com a densidade usada de fluxo no primeiro bloco de testes. O comportamento é similar ao descrito anteriormente para a penetração da solda e, em sentido oposto, para a largura do cordão. Observa-se um aumento na área de fusão na soldagem ATIG em relação à soldagem TIG de aproximadamente $100 \%$, sendo que está área parece atingir um 


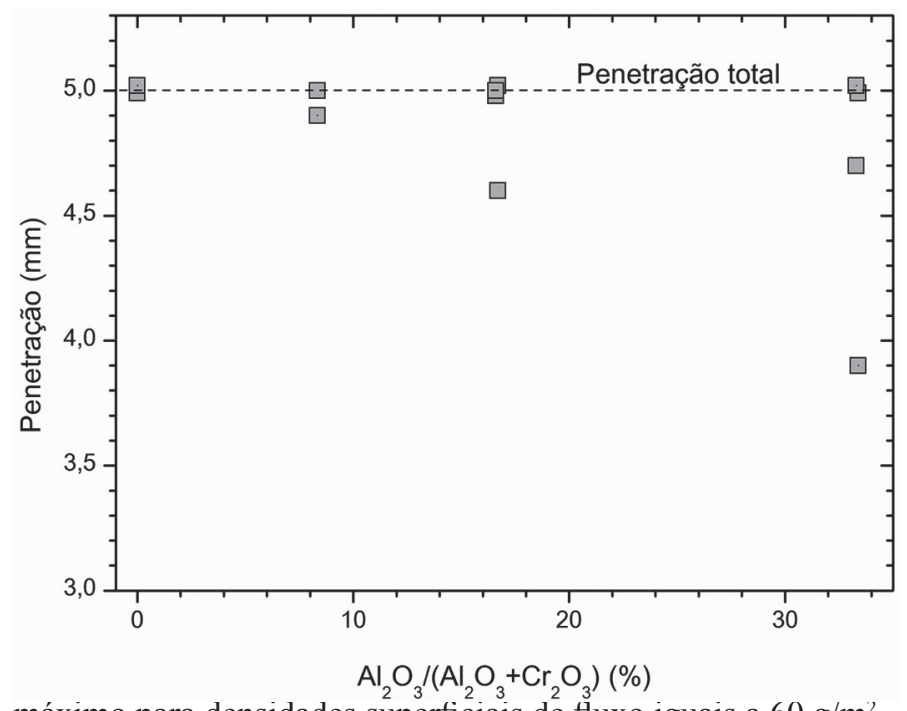

máximo para densidades superficiais de fluxo iguais a $60 \mathrm{~g} / \mathrm{m}^{2}$.

Figura 8. Variação da penetração do cordão em função da densidade superficial dos fluxos de $\mathrm{Cr}_{2} \mathrm{O}_{3}+\mathrm{Al}_{2} \mathrm{O}_{3}$.

O rendimento teórico de fusão na soldagem a arco pode ser definido como a razão entre a energia mínima necessária para aquecer e fundir a ZT e a energia gerada no arco, isto é:

$\eta_{f}=\frac{A_{f} \cdot v \cdot \rho \cdot\left(\Delta H_{\text {Aquec }}+\Delta H_{f}\right)}{U I}$

onde $A_{f}$ é a área de fusão, $v$ é a velocidade de soldagem, $r$ é a densidade do material, $\Delta H_{\text {Aquec }}$ e $\Delta H_{f}$ são, respectivamente, as quantidades de energia necessárias para o aquecimento e a fusão do material e $U$ e $I$ são a tensão e corrente de soldagem.

Como, nas condições de ensaio usadas neste trabalho, a velocidade, a tensão e a corrente praticamente não foram alteradas durante as soldagem TIG e ATIG (exceto por um aumento inferior a $1 \mathrm{~V}$ na tensão de soldagem) e que o mesmo material foi sempre usado, o rendimento teórico pode ser considerado como diretamente proporcional à área da seção transversal da solda. Assim, os resultados obtidos indicam um aumento de cerca de $100 \%$ no rendimento teórico de fusão do processo quando se compara as soldagens TIG e ATIG. Utilizando, na equação (2), valores do calor específico do aço inoxidável 316 em função da temperatura [24], o calor de fusão do ferro $(245 \mathrm{~J} / \mathrm{g})$, a densidade típica de um aço inoxidável austenítico $\left(8,0 \mathrm{~g} / \mathrm{cm}^{3}\right)$ e os valores obtidos de $A_{f}, v, U$ e $I$ nos testes realizados, obtém-se, para as soldagens TIG e ATIG, valores de rendimento teórico de aproximadamente 15 e $30 \%$, respectivamente. Na soldagem a arco, o rendimento teórico de fusão varia tipicamente entre cerca de 5 e $50 \%$ [25], assim, o aumento observado neste rendimento para a soldagem ATIG pode ser considerado bastante expressivo.

Os valores da área de fusão obtidos no $1^{\circ}$ bloco (fluxo: $\mathrm{Cr}_{2} \mathrm{O}_{3}$ ) e no $2^{\circ}$ bloco (fluxo: $\mathrm{Cr}_{2} \mathrm{O}_{3}+\mathrm{KClO}_{4}$ ) de testes são comparados na figura 10. Estes resultados mostram que, além da redução na penetração, também ocorre uma redução da capacidade de fusão do processo com a adição do perclorato de potássio. Este efeito é mais claro para as duas maiores densidades de fluxo usadas no $2^{\circ}$ bloco de testes $\left(30\right.$ e $\left.60 \mathrm{~g} / \mathrm{m}^{2}\right)$.

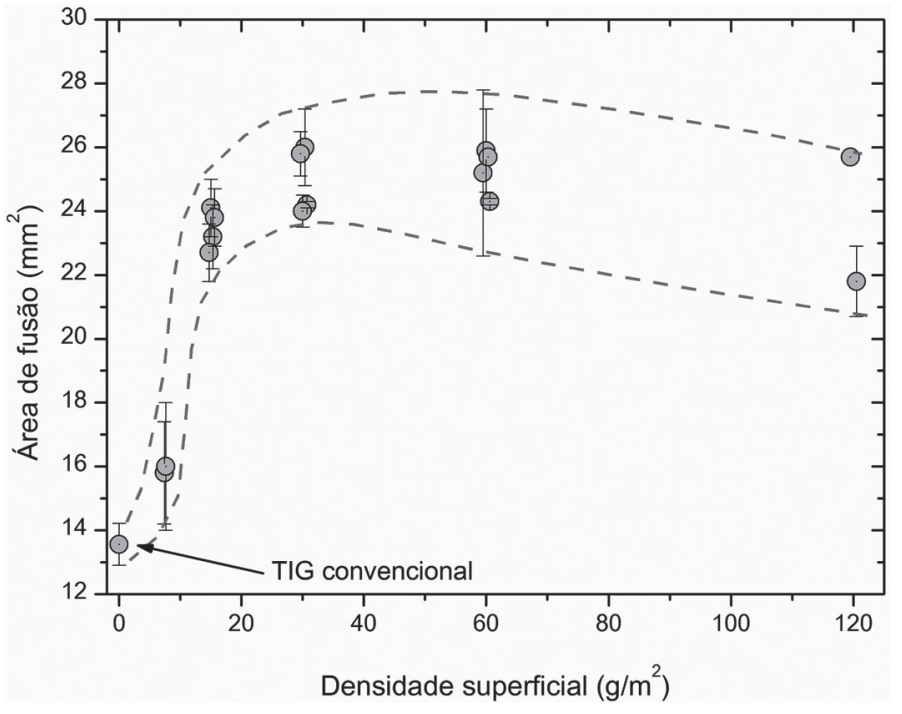

Figura 9. Variação da área da seção transversal do cordão em função da densidade superficial dos fluxos de $\mathrm{Cr}_{2} \mathrm{O}_{3}$.

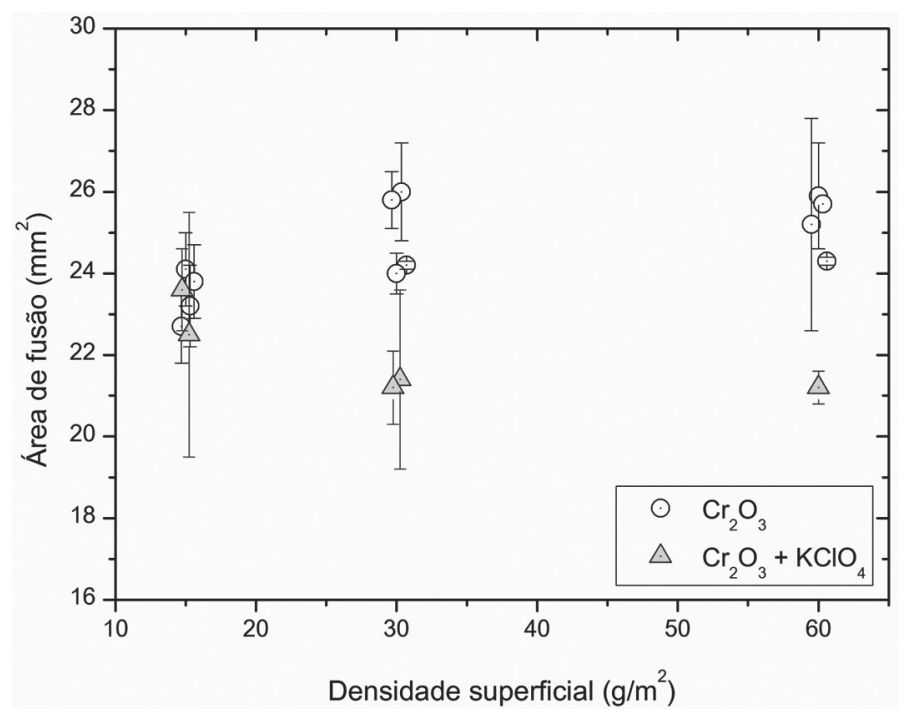

Figura 10. Variação da área da seção transversal do cordão em função da densidade superficial dos fluxos de $\mathrm{Cr}_{2} \mathrm{O}_{3}$ e de $\mathrm{Cr}_{2} \mathrm{O}_{3}$ $+\mathrm{KClO}_{4}$.

O efeito negativo da adição de perclorato de potássio no desempenho do fluxo a base de $\mathrm{Cr}_{2} \mathrm{O}_{3}$ foi, inicialmente, considerado inesperado tendo em vista o caráter fortemente oxidante deste composto. Este teria o potencial de, mesmo presente em pequenas quantidades, fornecer um maior volume de oxigênio para a poça de fusão e, assim, favorecer um aumento da penetração do cordão de solda ou possibilitar uma redução da quantidade de fluxo a ser usada. Considerando a reação de decomposição do perclorato de potássio (equação 1), observa-se que um mol deste composto $(138,5 \mathrm{~g})$ pode fornecer até quatro moles de oxigênio atômico ([O]) ao metal líquido.

Por outro lado, a decomposição do $\mathrm{KClO}_{4}$ ocorre em temperaturas relativamente baixas (em torno de 400 a $600^{\circ} \mathrm{C}$ ) e muito menores do que a faixa de temperatura de fusão do aço inoxidável 304. Desta forma, o oxigênio deve ser liberado na 
atmosfera de proteção e direcionado para longe da poça pelo jato de plasma e pelo próprio gás de proteção e não contribuir para elevar o teor de oxigênio na poça de fusão. Ao final da decomposição, o produto que resta é um cloreto $(\mathrm{KCl})$. Os resultados aqui obtidos sugerem que este não contribui para a constrição do arco, de modo similar ao atribuído para fluoretos (ver, por exemplo, com Leconte e colaboradores [26]), nem para afetar o movimento de metal líquido na poça de fusão. Assim, nos testes com o fluxo de $\mathrm{KClO}_{4}+\mathrm{Cr}_{2} \mathrm{O}_{3}$ foram observados indícios de uma menor capacidade de penetração da solda (em comparação com o fluxo simples de $\mathrm{Cr}_{2} \mathrm{O}_{3}$ ). Esta redução poderia ser causada pela diluição do $\mathrm{Cr}_{2} \mathrm{O}_{3}$ no fluxo com o $\mathrm{KCl}$, contudo, como este efeito foi observado mesmo para uma densidade superficial de $60 \mathrm{~g} / \mathrm{m}^{2}$, alguma interação entre os dois composto, além de uma simples mistura, deve ter ocorrido.

$\mathrm{O} \mathrm{Cr}_{2} \mathrm{O}_{3}$ apresenta uma estabilidade muito superior ao $\mathrm{KClO}_{4}$. Em temperaturas elevadas e em contato com atmosferas com potencial de oxigênio muito baixo (Figura 11), esse óxido deve se decompor, por exemplo, de acordo com a equação (3), podendo, assim, liberar oxigênio para a poça de fusão.

$\mathrm{Cr}_{2} \mathrm{O}_{3} \rightleftarrows 2 \mathrm{CrO}+\underline{\mathrm{O}}$

Medidas por termogravimetria durante a sinterização de pó de $\mathrm{Cr}_{2} \mathrm{O}_{3}$ indicam perda de massa a partir de $1470^{\circ} \mathrm{C}$ pela dissociação deste óxido e a formação de $\mathrm{CrO}$ no estado líquido [27]. Estas temperaturas permitem que a liberação de oxigênio ocorra junta da poça de fusão e, assim, possa influenciar o movimento do metal líquido nesta.

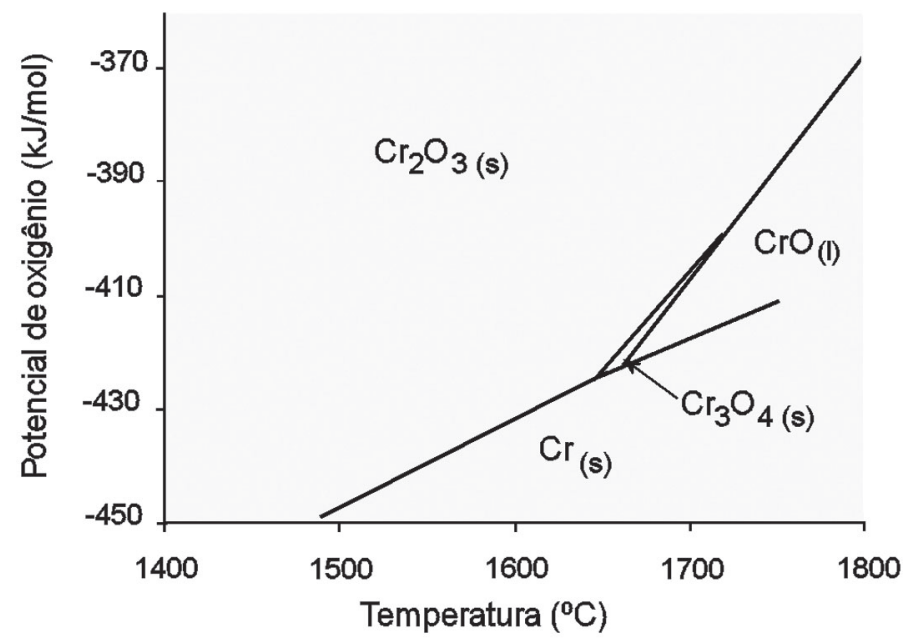

Figura 11. Diagrama termodinâmico do sistema Cr-O (Adaptado de [27]).

\section{Conclusões}

No presente trabalho foi feito um estudo da influência da densidade de um fluxo simples de $\mathrm{Cr}_{2} \mathrm{O}_{3}$ espalhado na superfície do corpo de prova e da presença de dois aditivos $\left(\mathrm{Al}_{2} \mathrm{O}_{3}\right.$ e $\left.\mathrm{KClO}_{4}\right)$ neste fluxo sobre as características operacionais e a geometria do cordão de solda obtido com o processo de soldagem ATIG. As principais conclusões deste estudo foram:
- Nas condições de soldagem usadas, o fluxo de um componente $\left(\mathrm{Cr}_{2} \mathrm{O}_{3}\right)$ possibilitou a soldagem com penetração total em chapas de aço inoxidável ABNT 304 com $5 \mathrm{~mm}$ de espessura para densidades superficiais deste fluxo entre 15 e $60 \mathrm{~g} / \mathrm{m}^{2}$.

- Para uma densidade menor, não foi obtida penetração total para as condições de soldagem usadas. Para uma densidade igual a $120 \mathrm{~g} / \mathrm{m}^{2}$, embora tenha sido possível obter penetração total na solda, observaram-se indícios de uma menor capacidade de penetração.

- Associado o rendimento teórico de fusão do processo TIG com a área da seção transversal do cordão foi possível mostrar um aumento de aproximadamente $100 \%$ neste rendimento com a aplicação superficial do fluxo simples.

- A adição de $\mathrm{KClO}_{4}$, embora este seja um componente de elevado poder de oxidação, reduziu a capacidade de penetração do cordão na soldagem ATIG com fluxo a base de $\mathrm{Cr}_{2} \mathrm{O}_{3}$.

- Atribuiu-se esta redução a dois fatores: (a) a liberação de oxigênio pelo perclorato de potássio a temperaturas muito baixas e inferiores à temperatura de início de fusão do metal base e (b) a uma diluição do óxido de cromo pelo aditivo, resultando em uma menor atividade do fluxo.

- A adição de $\mathrm{Al}_{2} \mathrm{O}_{3}$, componente que, quando usado individualmente não causa aumento de penetração na soldagem ATIG, também reduziu a capacidade de penetração do cordão na soldagem com fluxo a base de $\mathrm{Cr}_{2} \mathrm{O}_{3}$. Neste caso, o resultado parece ser causado por um efeito de diluição do fluxo.

\section{Agradecimentos}

Os autores agradecem à APERAM SOUTH AMERICA e à ESAB Comércio e Indústria Ltda pelo fornecimento de materiais usados neste estudo e, ao Conselho Nacional de Desenvolvimento Científico e Tecnológico (CNPq), pelo suporte para a realização deste projeto.

\section{Referências Bibliográficas}

[1] LUCAS, W., HOWSE, D., Activating flux-increasing the performance and productivity of the TIG and Plasma processes. Welding \& Metal Fabrication, p.11-17, Jan. 1996.

[2] SHAMPING LU, HIDETOSHI FUJI, KIYOSHI NOGI, Weld Shape Variation and Electrode Oxidation Behavior under Ar-(Ar-CO2) Double Shielded GTA Welding, J. Mater. Sci. Technol., v. 26, n. 2, p. 170-176, 2010.

[3] MESSLER JR., R. W., Principles of Welding, John Wiley \& Sons, 1999 , p. 56.

[4] HIDETOSHI FUJII, SHAMPING LU, TOYOYUKI SATO, KIYOSHI NOGI, Effect of oxygen content in He-O2 shielding gas on weld shape for ultra-deep penetration TIG, Transactions of JWRI, v. 37, n. 1, p. 19-26, 2008.

[5] LEINONEN, J. I., KARJALAINEN, L. P., Unexpected weld pool profiles in GTA welding with oxidizing shielding gas. In: RECENT TRENDS IN WELDING SCIENCE AND TECNOLOGY PROCEEDINGS, 2nd International Conference, Gatlinburg, May 1989, University of Oulu, Finland, p. 387-390. Eds.: 
DAVID S. A., VITEK J. M. Publ. Materials Park, USA; ASM International, p.14-18, 1990.

[6] ANDERSON, P. C. J., WIKTOROWICZ, R., Improving productivity with A-TIG welding, Welding \& Metal Fabrication, Março, p.108-109, 1996.

[7] MODENESI, P. J., APOLINÁRIO, E. R., PEREIRA, I. M., TIG Welding with Single-Component Fluxes, Journal of Materials Processing Technology, v. 99, p. 260-265, 2000.

[8] KAZAKOV, Y.V. et al., Effect of activating fluxes on the structure of the welding arc in argon. Svarochnoe Proizvodstvo, n. 4, 1991, p. 30-32, 1991.

[9] ZAMKOV, V. N., PRILUTSKII, V. P., GUREVICH, S. M. The effective ionization potential of the arc when titanium is welded by the semi-submerged arc process, Avtomaticheskaya Svarka, n.1, p.13-15, 1977.

[10] ZAMKOV, V. N., PRILUTSKII, V. P., GUREVICH, S. $M$. The effects of the composition of the flux on the process of welding titanium with a non-consumable electrode, Avtomaticheskaya Svarka, n.4, p.22-26, 1977.

[11] HIDETOSHI FUJI, TOYOYUKI SATO, SHAMPING LU, KIYOSHI NOGI, Development of an advanced A-TIG (AA-TIG) welding method by control of Marangoni convection, Materials Science and Engineering A, v. 495, p. 296-303, 2008. [12] XU, Y.L., DONG, Z.B., WEI, Y.H., YANG, C.L., Marangoni convection and weld shape variation in A-TIG welding process, Theoretical and Applied Fracture Mechanics, v. 48, p. 178-186, 2007.

[13] PERRY, N., Etude et développement des flux solides en vue d'application en soudage ATIG appliqué au titane et ses alliages ainsi qu'aux aciers inoxydables. Tese de doutorado, Laboratoire de Mécanique et Matériaux, ECOLE CENTRALE DE NANTES - ECN, 2000.

[14] LU, S., FUJII, H., TANAKA, M., NOGI, K. Oxide flux quantity and size effects on the penetration depth in A-TIG welding, Transactions of JWRI, v. 31, n. 2, p. 187-192, 2002.

[15] PERRY, N., MARYA, S., New perspectives of flux assisted GTA welding in titanium structures, p. 55-62, 1999. Obtido em: www.wahchanglabs.com/pdf/1999/1999009.pdf em janeiro de 2009.

[16] SHYU, S. W, HUANG, H. Y., TSENG, K.H., CHOU, C. P., Study of the Performance of Stainless Steel A-TIG Welds, JMEPEG, v. 17, p.193-201, 2008.

[17] KUANG-HUNG TSENG, CHIH-YU HSU, Performance of activated TIG process in austenitic stainless steel welds, Journal of Materials Processing Technology 211, 503-512, 2011

[18] LI QING-MING, WANG XIN-HONG, ZOU ZENG-DA, WU JUN, Effect of activating flux on arc shape and arc voltage in tungsten inert gas welding - Trans. Nonferrous Met. Soc. China I7, 486-490, 2007.

[19] H. Y. HUANG, S. W. SHYU, K. H. TSENG, C. P. CHOU, Evaluation of TIG flux welding on the characteristics of stainless steel, Science and Technology of Welding and Joining, 10 (5), 566-573, 2005.

[20] ZHANG, R. H., FAN, D., Numerical simulation of effects of activating flux on flow patterns and weld penetration in ATIG welding, Science and Technology of Welding and Joining, 12 (1) $15-23,2007$
[21] DONG, C., ZHU, Y., CHAI, G., ZHANG, H., Preliminary study on the mechanism of arc welding with the activating flux. 8p. Obtido, em janeiro de 2010 do endereço:

http://scholar.ilib.cn/Article.aspx?AIT $=\mathrm{QCode} \& \mathrm{AI}=\mathrm{hkgyjs} 200$ 4z1072\&A=hkgyjs2004z1072

[22] HER-YUEH HUANG, Argon-Hydrogen Shielding Gas Mixtures for Activating Flux-Assisted Gas Tungsten Arc Welding, Metallurgical and Materials Transactions A, v. 41A, p. 2829-2835, Nov. 2010.

[23] LU, S., FUJII, H., SUGIYAMA, H., TANAKA, M., NOGI, $\mathrm{K}$., Marangoni convection and welding penetration in A-TIG welding, Transactions of JWRI, v. 32, n. 1, p. 79-82, 2003.

[24] REDMOND, R. F., LONES, J., Enthalpies and heat capacities of stainless steel (316), zirconium, and lithium at elevated temperatures, Relatório Técnico, Oak Ridge National Laboratory, $24 \mathrm{p} ., 1952$

[25] DUPONT, J. N., MARDER, A. R., Thermal efficiency of arc welding processes, Welding Journal, December, p. 406s416s, 1995.

[26] LECONTE, S., PAILlARD, P., CHAPELLE, P., HENRION, G., SAINDRENAN, J., Effects of flux containing fluorides on TIG welding process, Science and Technology of Welding and Joining, vol. 12 n. 2, p.120-126, 2007.

[27] PERES, V., FAVERGEON, L., ANDRIEU, M., PALUSSIĖRE, J.-C., BALLAND, J., DELAFOY, C., PIJOLAT, M., High temperature chromium volatization from $\mathrm{Cr}_{2} \mathrm{O}_{3}$ powder and $\mathrm{Cr}_{2} \mathrm{O}_{3}$-doped $\mathrm{UO}_{2}$ pellets in reducing atmospheres, Journal of Nuclear Materials, 423(1-2), 93-101, 2012. 